\title{
Molecular epidemiology of Mycobacterium tuberculosis in East Lancashire 2001-2009
}

\author{
A D Sails, ${ }^{1}$ A Barrett, ${ }^{1}$ S Sarginson, ${ }^{1}$ J G Magee, ${ }^{1}$ P Maynard, ${ }^{2}$ I Hafeez, ${ }^{3}$ \\ L P Ormerod ${ }^{3,4}$
}

${ }^{1}$ HPA Regional Centre for Mycobacteriology, Newcastle General Hospital, Newcastle upon Tyne, UK ${ }^{2}$ Department of Microbiology, Royal Blackburn Hospital, Blackburn, Lancashire, UK ${ }^{3}$ Department of Respiratory Medicine, Royal Blackburn Hospital, Blackburn, Lancashire, UK

${ }^{4}$ Lancashire Postgraduate School of Medicine, University of Central Lancashire, Preston, Lancashire, UK

\section{Correspondence to}

Professor L P Ormerod, Royal Blackburn Hospital, Blackburn, Lancashire BB2 3HH, UK; lawrence.ormerod@elht.nhs.uk

Received 4 January 2011 Accepted 28 April 2011 Published Online First 15 June 2011

\section{ABSTRACT \\ Background East Lancashire has had high rates of tuberculosis for 40 years. The ethnically diverse population is predominantly of South Asian and white origin. Drug resistance data from 1960 to 1999 indirectly suggest that no significant inter-ethnic transmission has occurred. This study used mycobacterial interspersed repetitive unit variable number tandem repeat (MIRU- VNTR) fingerprinting to assess clustering within and between ethnic groups.}

Methods All isolates of Mycobacterium tuberculosis from January 2001 to July 2009 from East Lancashire postcode areas were MIRU-VNTR fingerprinted. Clusters of strains with indistinguishable profiles were also assessed epidemiologically, and their MIRU-VNTR profiles compared with the UK M tuberculosis Strain Typing Database.

Results 332 strains were typed (63 white patients, and 269 non-white patients). 198 MIRU-VNTR profiles were identified, with 144 profiles occurring only once. The typing clustered 187 strains into 53 clusters indistinguishable at all 12 loci and these were further characterised using the exact tandem repeat loci $A, B$, and $\mathrm{C}$. The 15 loci clustered 32/63 (50.8\%) of white and $110 / 269(40.9 \%)$ of non-white cases and all but nine clusters were of the same ethnicity. The nine inter-racial clusters were further assessed from an epidemiological and clinical perspective and fingerprinting using nine additional loci. Isolates within two of the clusters were further discriminated using the additional nine loci. However, the additional loci did not further discriminate the isolates in the other seven inter-racial clusters. Conclusions MIRU-VNTR fingerprinting indicates that although there is evidence of a high rate of transmission within the South Asian sub-population, the data suggest that there is little inter-ethnic transmission.

\section{INTRODUCTION}

Blackburn was in the top 10 tuberculosis (TB) incidence local government areas of England and Wales in 1978-9, ${ }^{1}$ and remained in the top 20 incidence areas through to $1998 .^{23}$ The Pendle area of East Lancashire was also consistently in the top 20 incidence areas over this same time period. ${ }^{1-3}$ In 2006-8 the incidence of TB in Blackburn averaged $37 / 100000$ per annum and that for East Lancashire, including Pendle, 16/100 000 per annum. ${ }^{4}$ Detailed epidemiological data on TB drug resistance have been reported for the Blackburn, Hyndburn, and Ribble Valley parts of East Lancashire for a 40 year timescale. Sequential surveys of drug resistance in both the white and South Asian ethnic groups for 1960-85, ${ }^{5} 1986-90,{ }^{6}$ and $1991-2000^{7}$ showed

\section{Key messages}

What is the key question?

- Is there evidence of significant tuberculosis transmission between the main ethnic groups in East Lancashire?

What is the bottom line?

- There is little evidence of inter-racial transmission, but over $50 \%$ of white cases are recently acquired as are a minimum of $19 \%$ of South Asian cases.

Why read on?

- The data demonstrate the usefulness of mycobacterial interspersed repetitive unit variable number tandem repeat (MIRU-VNTR) fingerprinting in helping with local epidemiology and potential sites of transmission.

rapidly reducing rates of initial drug resistance in the white population from 1960 to 1975 with very low rates since then. However, a consistently high rate $(7-10 \%)$ of initial isoniazid resistance occurred in the South Asian ethnic population throughout the period 1960-2000 inclusive. ${ }^{5-7}$ This has provided strong indirect evidence of the lack of transmission of TB between the South Asian and white ethnic groups in this area. The development of molecular typing methods for strains of $\mathrm{Myco}$ bacterium tuberculosis facilitates fingerprinting of $\mathrm{TB}$ isolates to provide more direct, as opposed to indirect, evidence for transmission of $\mathrm{TB}$ between and within different ethnic groups.

The sequencing of the entire genome of a number of bacterial pathogens has revealed the presence of variable number tandem repeats (VNTRs) in bacterial genomes which appear analogous to human micro-satellites. The variability in repeat number of VNTR loci can be exploited as a method of fingerprinting these pathogens. The first method described for the VNTR typing of $M$ tuberculosis targeted five exact tandem repeat loci (ETR) A, B, $\mathrm{C}, \mathrm{D}$, and $\mathrm{E}^{8}$ This PCR-based method, while easily standardised between laboratories to facilitate data comparison, did however lack discriminatory power. An additional 10 VNTR loci specific to mycobacteria termed mycobacterial interspersed repetitive units (MIRUs) are targeted in the VNTR typing method known as MIRU-VNTR typing. Twelve VNTR loci are targeted in the MIRU-VNTR fingerprinting method including ETR D and E from the original Frothingham and Meeker-O'Connell 
scheme. $^{8}$ Isolates are fingerprinted based on the number of repeats at the 12 MIRU-VNTR loci located throughout the genome. An evaluation of the 12 MIRU loci and three ETR loci has previously demonstrated that they provide a similar level of discrimination as IS-6110 typing. ${ }^{10}$ An optimised set of 24 MIRU-VNTR loci has recently been defined for the typing of $M$ tuberculosis, which provides the highest level of discrimination between stains. ${ }^{11}$

\section{METHODS}

All isolates of $M$ tuberculosis from January 2001 to July 2009 inclusive from East Lancashire (Burnley, Pendle, Rossendale (BPR), and Blackburn, Hyndburn and Ribble Valley (BHRV) districts) were sent to the HPA Regional Centre for Mycobacteriology at Newcastle for identification and drug susceptibility testing. Isolates were grown in Mycobacterial Growth Indicator tubes (Becton Dickinson, UK). DNA was extracted from a $250 \mu \mathrm{l}$ aliquot of the culture using a OiaAmp DNA Blood Mini Kit (Qiagen Ltd, Crawley, West Sussex, UK) according to the manufacturer's protocol and the DNA was used as a template in the MIRU PCR reactions. After amplification, each PCR product was analysed on a WAVE denaturing high-performance liquid chromatography (DHPLC) system with the fragment size being calculated from the chromatogram, and the number of tandem repeats at each locus was determined as previously described. ${ }^{12}$ All of the 332 strains were characterised using the 12 MIRU-VNTR loci previously described. ${ }^{9}$ Strains which clustered into groups with indistinguishable profiles were further characterised using the ETR loci A, B and C. Strains which clustered into inter-racial groups that were indistinguishable at all 15 loci were further characterised using the nine additional MIRU-VNTR loci used for enhanced discrimination. ${ }^{11}$ Clusters of strains with indistinguishable profiles were also assessed epidemiologically by the clinicians and TB nurses, and had their MIRU-VNTR profiles compared with those in the UK $M$ tuberculosis Strain Typing Database containing MIRU-VNTR profiles of over 25000 isolates. $^{13}$

\section{RESULTS}

A total of 332 strains isolated between January 2001 and July 2009 were tested, with 63 obtained from white ethnic patients, and 269 from non-white patients. The majority of non-white patients were of South Asian ethnic origin $(n=262)$ the other seven being black African $(n=2)$, Chinese ethnic origin $(n=2)$, Indonesian $(n=1)$, Afghan $(n=1)$ and Phillipino $(n=1)$. MIRUVNTR fingerprinting using 12 loci differentiated the 332 strains into 198 distinct MIRU-VNTR profiles, with 144 profiles occurring only once in the data set. The 12 loci typing clustered 187 isolates into 53 clusters containing between two and 25 isolates which were indistinguishable from each other at all 12 MIRU-VNTR loci. The majority of the strains in these 53 clusters (182 of 187 strains) were further investigated by characterising the ETR A, B, and C loci if sufficient DNA extract was available. MIRU-VNTR fingerprinting with the 15 loci identified 42 groups of strains containing between two and 13 isolates which were indistinguishable from each other at all 15 MIRUVNTR loci (table 1). Five clusters identified using 12 loci typing each containing two isolates could not be confirmed using the ETR loci because no DNA extract was left for analysis for one of the two strains in each cluster. These clusters (4, 5, 16, 29, 34, 43) are included in table 1 because they were indistinguishable at 12 MIRU-VNTR loci. MIRU-VNTR analysis with 15 loci clustered $32 / 63$ (50.8\%) of white isolates and 110/269 (40.9\%) of non-white cases, with 142/332 (42.8\%) being clustered overall. Thirty-nine of the 48 clusters presented in table 1 were made up of strains from patients who were of the same ethnicity. Clusters of strains with indistinguishable profiles had their MIRUVNTR profiles compared with those in the UK $M$ tuberculosis Strain Typing Database to ascertain the frequency of these profiles in the UK (table 1). Results of typing the strains in the nine inter-racial clusters using the additional nine MIRU-VNTR loci are presented in table 2. Only 27 of the 30 strains could be tested because there was insufficient DNA available for three of the strains. The additional nine loci provided further discrimination of strains in clusters 9 and 17, indicating that the cases were not linked. However, fingerprinting with the additional nine loci identified that the strains in the other seven inter-racial clusters remained indistinguishable within each cluster. The nine possible inter-racial clusters were further assessed from an epidemiological and clinical perspective (table 2). Further epidemiological investigation of cluster 15 showed likely transmission from a South Asian college student to his flat mate and later to a white student with whom he had limited contact at college. Further epidemiological investigation of the other eight clusters showed no known epidemiological contact between white and South Asian cases.

\section{DISCUSSION}

This study was initiated in 2006, and then expanded with 2009 data, to measure the extent of potential clustering in our population, which continues to have high rates of $\mathrm{TB}$, largely in our main ethnic minority group, those of South Asian descent. ${ }^{14}$ Drug resistance data reported for part of our population from 1960 to $2000^{5-7}$ has shown widely divergent recent isoniazid resistance rates for the white $(0.5 \%<)$ and South Asian $(7-10 \%)$ isolates, suggesting little inter-racial transmission. In this study the isolates from our white ethnic group showed $51 \%$ potential clustering. A substantial proportion of this is supported by local epidemiology and contact data, which confirms most of the clustered cases link with either certain public houses (clusters 7-8, 10-11: 17 individuals), with a cohort of drug and/or alcohol users (cluster 2: eight individuals), or with ongoing clinical cases from 2001 to 2009. Two other individuals in cluster 9 and one individual in cluster 15 were also linked by epidemiology, making 28/63 (44\%) isolates definitely clustered, the other seven individuals in inter-racial clusters being less certainly linked.

The potential clustering rate was $41 \%$ for those of South Asian ethnicity. However when the MIRU-VNTR profiles of those strains which were clustered were compared with profiles in the UK Strain Typing Database, many of the profiles were shown to be common in the UK, for example clusters $2,9,15$, $17,25,31,33$ and 38 , with over 100 isolates previously identified nationally to have these profiles, and clusters 4 and 23, with over 300 isolates previously identified. Some of these apparently clustered cases may therefore be no more than the expression of a common profile in the population, and do not imply recent local transmission. Local recent transmission however is confirmed by epidemiological links in some South Asian clusters (18 and 28: four individuals), and in others by their MIRUVNTR profile. A considerable number of small local clusters (14, 19, 37, 41-42, 44-46, 48: 24 individuals) have unique profiles which have not been previously recognised in the UK Strain Typing Database, which is highly suggestive of recent local transmission. There are also a number of other clusters (3, 12-13, 24, 29-30, 32, 39, 43 and 47: 26 individuals) with MIRU-VNTR profiles which have occurred in fewer than 10 
Table 1 MIRU-VNTR profiles of clusters identified by MIRU-VNTR fingerprinting with 15 loci and their prevalence in the UK Mycobacterium tuberculosis Strain Typing Database (STD)

\begin{tabular}{|c|c|c|c|c|c|c|c|}
\hline \multirow[b]{2}{*}{ Cluster } & \multirow[b]{2}{*}{ MIRU-VNTR profile } & \multicolumn{3}{|l|}{ ETR } & \multirow[b]{2}{*}{ Number of isolates } & \multirow[b]{2}{*}{ Comments } & \multirow[b]{2}{*}{ Prevalence in STD } \\
\hline & & $\overline{\mathbf{A}}$ & B & $\overline{\mathbf{C}}$ & & & \\
\hline $1^{*}$ & 222325153323 & 4 & 1 & 4 & 2 & & Unique profile not otherwise in STD \\
\hline $2^{*}$ & 223125153322 & 3 & 2 & 4 & 9 & & 118 matching isolates \\
\hline 3 & 223315153324 & 3 & 2 & 4 & 2 & Both South Asian & 6 matching isolates \\
\hline 4 & 223325153324 & 5 & 2 & 4 & 2 & Both South Asian & 312 matching isolates (12 loci) \\
\hline 5 & 223425173533 & NA & 2 & 4 & 2 & Both South Asian & 24 matching isolates (12 loci) \\
\hline 6 & 224125163324 & 3 & 2 & 3 & 2 & Both South Asian & 45 matching isolates \\
\hline 7 & 224325143323 & 2 & 2 & 3 & 7 & All white, linked to a pub & 1 matching isolate \\
\hline 8 & 224325143325 & 4 & 2 & 3 & 3 & All white, linked to a pub & 11 matching isolates \\
\hline $9 *$ & 224325153323 & 3 & 2 & 4 & 5 & & 161 matching isolates \\
\hline 10 & 224325163325 & 3 & 2 & 3 & 3 & All white, linked to a pub & 2 matching isolates \\
\hline 11 & 224325163325 & 4 & 2 & 3 & 4 & All white, linked to a pub & Unique profile not in STD \\
\hline 12 & 224326163326 & 2 & 2 & 4 & 2 & Both South Asian & 1 matching isolate \\
\hline 13 & 224425173323 & 4 & 2 & 2 & 3 & All South Asian & 9 matching isolates \\
\hline 14 & 224425173533 & 5 & 2 & 2 & 5 & & Unique profile not otherwise in STD \\
\hline $15^{*}$ & 225125113322 & 3 & 2 & 4 & 2 & & 174 matching isolates \\
\hline $16^{*}$ & 225225173533 & 3 & 2 & 2 & 2 & & 12 matching isolates (12 loci) \\
\hline $17^{*}$ & 225313153323 & 3 & 2 & 3 & 2 & & 151 matching isolates \\
\hline 18 & 225325133323 & 4 & 2 & 2 & 2 & South Asian family, linked & 38 matching isolates \\
\hline 19 & 225421163533 & 5 & 2 & 2 & 2 & Both South Asian & Unique profile not otherwise in STD \\
\hline 20 & 225425143533 & 4 & 2 & 2 & 2 & Both South Asian & 26 matching isolates \\
\hline 21 & 225425173531 & 4 & 2 & 2 & 4 & All South Asian & 24 matching isolates \\
\hline 22 & 225425173533 & 3 & 2 & 2 & 3 & All South Asian & 19 matching isolates \\
\hline 23 & 225425173533 & 4 & 2 & 2 & 13 & All South Asian & 426 matching isolates \\
\hline 24 & 225425173533 & 5 & 2 & 2 & 5 & All South Asian & 1 matching isolate \\
\hline $25^{*}$ & 225425173533 & Null & 2 & 2 & 2 & & †103 matching isolates (14 loci) \\
\hline 26 & 225425183533 & 4 & 2 & 2 & 2 & Both South Asian & 41 matching isolates \\
\hline 27 & 226225173533 & 4 & 2 & 2 & 2 & Both South Asian & 3 matching isolates \\
\hline 28 & 226425152523 & 5 & 2 & 2 & 2 & South Asian, family linked & Unique profile not otherwise in STD \\
\hline 29 & 226425163523 & 4 & 2 & 2 & 2 & Both South Asian & 3 matching isolates \\
\hline 30 & 226425173332 & 3 & 2 & 2 & 2 & Both South Asian & 1 matching isolate \\
\hline 31 & 226425173423 & 4 & 2 & 2 & 3 & All South Asian & 186 matching isolates \\
\hline 32 & 226425173423 & 5 & 2 & 2 & 3 & All South Asian & 3 matching isolates \\
\hline 33 & 226425173423 & 4 & 2 & 2 & 2 & Both South Asian & 186 matching isolates \\
\hline 34 & 226425173424 & 4 & 2 & 2 & 2 & Both South Asian & 32 matching isolates \\
\hline 35 & 226425173531 & 4 & 2 & 2 & 2 & Both South Asian & 26 matching isolates \\
\hline 36 & 226425173532 & 4 & 2 & 2 & 2 & Both South Asian & 38 matching isolates \\
\hline 37 & 226425173532 & 5 & 2 & 2 & 2 & Both South Asian & Unique profile not otherwise in STD \\
\hline 38 & 226425173533 & 4 & 2 & 2 & 5 & All South Asian & 183 matching isolates \\
\hline 39 & 226425173533 & 5 & 2 & 2 & 5 & All South Asian & 1 matching isolate \\
\hline $40 *$ & 226425183531 & 4 & 2 & 2 & 2 & & 7 matching isolates \\
\hline 41 & 227425173522 & 4 & 2 & 2 & 2 & Both South Asian & Unique profile not otherwise in STD \\
\hline 42 & 227425173532 & 4 & 2 & 2 & 5 & All South Asian & Unique profile not otherwise in STD \\
\hline 43 & 244326221533 & 10 & 4 & 4 & 2 & Both South Asian & 2 matching isolates \\
\hline 44 & 244327223426 & 6 & 1 & 4 & 2 & Both South Asian & Unique profile not otherwise in STD \\
\hline 45 & 254326223513 & 7 & NA & 2 & 2 & Both South Asian & Unique profile not otherwise in STD \\
\hline 46 & 254326223633 & 6 & 1 & 4 & 2 & Both South Asian & Unique profile not otherwise in STD \\
\hline $47^{*}$ & 274326223633 & 6 & 1 & 4 & 2 & & 2 matching isolates \\
\hline 48 & 274328223534 & 7 & 1 & 4 & 2 & Both South Asian & Unique profile not otherwise in STD \\
\hline
\end{tabular}

* Interracial clusters.

†Cluster 25 profile. 103 isolates in STD with the ETR A locus as a 'null' negative allele result. Appears to be a peculiarity of this strain. Clusters 4, 5, 16, 29, 34, and 43 are included because they were indistinguishable at 12 MIRU-VNTR loci. At the time of analysis (March 2010) there were 24869 records on the STD, from Newcastle RCM (3981), Birmingham RCM (7341), London MRU (7769), Scottish SRML (1598), Welsh Reference Lab (1526), and the London Study 1995-7 (2654).

ETR, exact tandem repeat; MIRU, mycobacterial interspersed repetitive unit; NA, no amplification; STD, Strain Typing Database; VNTR, variable number tandem repeat.

isolates in the UK Strain Typing Database, which contains nearly 25000 isolate profiles. Local transmission is therefore likely in these cases too. Those with proven or highly likely local transmission therefore account for at least 50/262 (19.1\%) of the South Asian cases. The 'true' clustering rate in the South Asian local population therefore lies somewhere between this $19.1 \%$ rate and the $43 \%$ overall clustering seen in the complete data.
The nine potential inter-racial clusters were investigated in more detail clinically, epidemiologically and by an additional nine more discriminatory MIRU-VNTR loci. Cluster 15 showed transmission in a college setting, but none of the other eight clusters showed any epidemiological link. Cluster 17 was separated by different drug susceptibility profiles and the isolates also differed at five of the nine additional loci. The additional nine loci further discriminated the five isolates in cluster 9 into 
Table 2 Patient characteristics, epidemiological links and results of enhanced mycobacterial interspersed repetitive unit (MIRU) (nine loci) typing for possible inter-racial clusters identified using 15 locus MIRU variable number tandem repeat (VNTR) typing

\begin{tabular}{|c|c|c|c|c|c|}
\hline Cluster number & Year of isolation & $\begin{array}{l}\text { Patient } \\
\text { characteristics (age) }\end{array}$ & $\begin{array}{l}\text { Site of isolation } \\
\text { (smear/culture result)* }\end{array}$ & Epidemiological links & $\begin{array}{l}\text { Enhanced MIRU typing } \\
\text { (nine loci) } \dagger\end{array}$ \\
\hline \multirow[t]{2}{*}{1} & 2003 & White female (65) & Peritoneum & \multirow[t]{2}{*}{ No known connection } & 222422353 \\
\hline & 2003 & Asian male (51) & Pulmonary $(S+)$ & & 222422353 \\
\hline \multirow[t]{9}{*}{2} & 2002 & Asian female (36) & Cervical gland & \multirow[t]{2}{*}{ No known connection } & Not tested $\ddagger$ \\
\hline & 2004 & White male (42) & Pulmonary $(S+)$ & & 224423542 \\
\hline & 2005 & White male (29) & Spinal disease & \multirow{7}{*}{$\begin{array}{l}\text { All linked by residence in hostel/and or } \\
\text { a group of alcohol and/or drug users }\end{array}$} & 224423542 \\
\hline & 2007 & White male (55) & Pulmonary $(S+)$ & & 224423542 \\
\hline & 2007 & White female (35) & Pulmonary $(\mathrm{S}+)$ & & 224423542 \\
\hline & 2007 & White female (24) & Pulmonary $(\mathrm{S}+)$ & & 224423542 \\
\hline & 2007 & White male (78) & Pulmonary $(\mathrm{C}+)$ & & 224423542 \\
\hline & 2009 & White male (37) & Pulmonary $(\mathrm{S}+)$ & & 224423542 \\
\hline & 2009 & White female (43) & Pulmonary $(\mathrm{S}+)$ & & 224423542 \\
\hline \multirow[t]{5}{*}{9} & 2005 & White male (25) & Pulmonary $(\mathrm{S}+)$ & \multirow[t]{5}{*}{ Cases 1,3 , and 4 linked socially } & 244443441 \\
\hline & 2006 & Asian male (33) & Pulmonary $(\mathrm{C}+)$ & & 434343312 \\
\hline & 2006 & White male (38) & Pulmonary $(\mathrm{S}+)$ & & 244413341 \\
\hline & 2008 & White female (49) & Pulmonary $(S+)$ & & 244413343 \\
\hline & 2008 & White male (33) & Pulmonary $(S+)$ & & 244443441 \\
\hline \multirow[t]{3}{*}{15} & 2004 & Asian male (30) & Pulmonary $(\mathrm{S}+)$ & \multirow{3}{*}{$\begin{array}{l}\text { Both Asian males shared accommodation } \\
\text { and went to same college. Case } 3 \text { shared } \\
\text { some classes }\end{array}$} & 132443383 \\
\hline & 2004 & Asian male (43) & Pulmonary $(\mathrm{S}+)$ & & 132443383 \\
\hline & 2005 & White male (18) & Pulmonary $(\mathrm{S}+)$ & & 132443383 \\
\hline \multirow[t]{2}{*}{16} & 2001 & White male (35) & Pulmonary $(S+)$ & \multirow[t]{2}{*}{ No known connection } & Not tested \\
\hline & 2004 & Asian female (58) & Cervical gland & & 44242337A§ \\
\hline \multirow[t]{2}{*}{17} & 2004 & White male (73) & Pulmonary $(\mathrm{S}+)$ & \multirow{2}{*}{$\begin{array}{l}\text { Not connected. Asian case isoniazid } \\
\text { mono-resistance, white case fully } \\
\text { susceptible. }\end{array}$} & 135243573 \\
\hline & 2004 & Asian female (36) & Dactylitis & & 226243172 \\
\hline \multirow[t]{3}{*}{25} & 2002 & Asian male (33) & Pulmonary (S+) & \multirow[t]{3}{*}{ No known connection } & 563423384 \\
\hline & 2002 & White male (49) & Pulmonary $(\mathrm{C}+)$ & & Not tested \\
\hline & 2004 & Asian male (18) & Cervical gland & & $-4-423384$ \\
\hline \multirow[t]{2}{*}{40} & 2006 & Asian female (19) & Pulmonary (S+) & \multirow[t]{2}{*}{ No known connection } & 442423384 \\
\hline & 2007 & White male (74) & Pulmonary $(\mathrm{S}+)$ & & 442423384 \\
\hline \multirow[t]{2}{*}{47} & 2004 & White male (70) & Cervical gland & \multirow[t]{2}{*}{ No known connection } & $2-4313461$ \\
\hline & 2004 & Asian male (43) & Ankle & & $2-4313461$ \\
\hline
\end{tabular}

NB. All cases with non-respiratory disease had normal chest x-rays.

*Smear/culture result: $\mathrm{S}+$, sputum microscopy positive; $\mathrm{C}+$, sputum microscopy negative, culture positive.

†Enhanced MIRU typing: MIRU loci 424, 1955, 2163b, 2347, 2401, 3171, 3690, 4052, 4156.

$\ddagger$ Not tested: insufficient DNA extract to perform additional typing; -, no PCR amplification for this locus.

$\S A=10$ repeats.

four different profiles, demonstrating that only two strains were indistinguishable. Of the other six profiles, cluster 47 was all non-pulmonary with no known association. The remaining clusters suggest if there was a possible direction of infection from a combination of clinical type, particularly sputum microscopy positive disease, and timing of isolates, that this was no more common for South Asian to white (clusters 1, 15, 25,40), than white to South Asian (clusters 2, 16).

Our overall rate of potential clustering (42.7\%) is higher than that reported in England and Wales, using an earlier technology, restriction-fragment-length-polymorphism analysis, at $22.7 \%$ clustering in 1995-7. ${ }^{15}$ Using spoligotyping and hemi-nestedinverse PCR this was a little higher at $27 \%$ in London in $2002 .{ }^{16}$ It is however within the range of clustering shown in cities across Europe, ranging from $30-35 \%$ in Brussels ${ }^{11}$ and Hamburg $^{17}$ to $53-61 \%$ in Spain $^{18}$ and Lisbon. ${ }^{19}$

This study has confirmed a high local transmission rate, particularly in subgroups of our white population, and shown the importance of public house related transmission ${ }^{20}$ in these groups, and also enabling targeted education and surveillance to be performed to try and break the cycle. Additionally, the data suggest that there is little inter-ethnic transmission, particularly from the South Asian population to the white ethnic group. Finally it has helped us recognise outbreaks and make decisions on extended contact tracing. The results of this study are directly relevant to other areas where TB has a high prevalence and an ethnically diverse population.

As this MIRU-VNTR technology is expanded to 24 loci, and becomes more 'real-time', it will be of increasing clinical relevance in managing patients with TB and contact investigations.

Acknowledgements We would like to thank the Cumbria and Lancashire Health Protection Unit for funding the retrieval and typing of the 2001-4 TB isolates.

Funding Other Funders: Health Protection Agency (2001-4 data).

Provenance and peer review Not commissioned; externally peer reviewed.

\section{REFERENCES}

1. Medical Research Council Tuberculosis and Chest Diseases Unit. Geographical distribution of tuberculosis notifications in a national survey of England and Wales (1978-9). Tubercle 1982;62:75-88.

2. Ormerod LP, Charlett A, Gillham C, et al. Geographical distribution of tuberculosis notifications in national surveys of England and Wales in 1988 and 1993. Thorax 1998; 53:176-81.

3. Rose AM, Watson JM, Graham C, et al. Tuberculosis at the end of the 20th century in England and Wales: results of a national survey in 1998. Thorax 2001;56:173-9.

4. Annual report on tuberculosis surveillance in the United Kingdom 2009. London: Health Protection Agency Centre for Infections, 2009.

5. Ormerod LP, Harrison JM, Wright PA. Drug resistance in $M$. tuberculosis: a survey over 25 years in Blackburn 1960-84. Thorax 1986;41:946-50.

6. Ormerod LP, Harrison JM, Wright PA. Drug resistance trends in Mycobacterium tuberculosis: Blackburn 1985-89. Tubercle 1990;71:283-5.

7. Ormerod LP, Green RM, Horsfield N, et al. Drug resistance trends in M. tuberculosis: Blackburn 1990-99. Int J Tuberc Lung Dis 2001:5:903-5. 
8. Frothingham R, Meeker-0'Connell WA. Genetic diversity in the Mycobacterium tuberculosis complex based on variable numbers of DNA repeats. Microbiology 1998;144:1189-96.

9. Supply $\mathbf{P}$, Lesjean E, Savine E, et al. Automated high-throughput genotyping for study of global epidemiology of Mycobacterium tuberculosis based on mycobacterial interspersed repetitive units. J Clin Microbiol 2001;39:3563-71.

10. Hawkey PM, Smith EG, Evans JT. Mycobacterial interspersed repetitive unit typing of Mycobacterium tuberculosis compared to IS6110 based restriction fragment length polymorphism analysis for investigation of apparently clustered cases of tuberculosis. J Clin Microbiol 2003;41:3514-20.

11. Oelemann MC, Diel R, Vatin V, et al. Assessment of an optimized mycobacterial interspersed repetitive-unit-variable-number tandem-repeat typing system combined with spoligotyping for population-based molecular epidemiology studies of tuberculosis. J Clin Microbiol 2007;45:691-7.

12. Evans JT, Hawkey PM, Smith EG, et al. Automated high-throughput mycobacterial interspersed repetitive unit typing of Mycobacterium tuberculosisstrains by a combination of PCR and nondenaturing high-performance liquid chromatography. J Clin Microbiol 2004;42:4175-80.

13. UK Mycobacterium tuberculosis Strain Typing Database. http://www.hpa-bionum.org. uk/TBtyping/home.php (accessed Mar 2010).
14. Tuberculosis in the UK: annual report on TB surveillance in the United Kingdom 2010 London: Health Protection Agency Centre for Infections, 2010.

15. Maguire H, Dale J, McHugh T, et al. Molecular epidemiology of tuberculosis in London 1995-7 showing low rate of active transmission. Thorax 2002; 57:617-22

16. Hayward AC, Goss S, Drobniewski F, et al. The molecular epidemiology of tuberculosis in Inner London. Epidemiol Infect 2002;128:175-84.

17. Allix-Beguec C, Fauville-Dufaux M, Supply P. Three-year population based evaluation of standardized mycobacterial interspersed repetitive-unit-variable number tandem-repeat typing of Mycobacterium tuberculosis. J Clin Microbiol 2008; $46: 1396-406$.

18. López-Calleja Al, Lezcano MA, Vitoria MA, et al. Genotyping of Mycobacterium tuberculosis over two periods: a changing scenario for tuberculosis transmission. Int J Tuberc Lung Dis 2007;11:1080-6.

19. Perdigão J, Macedo R, João I, et al. Multidrug-resistant tuberculosis in Lisbon, Portugal: a molecular epidemiological perspective. Microb Drug Resist 2008:14:133-43

20. De Vries G, van Hest RA, Burdo CC, et al. A Mycobacterium tuberculosis cluster demonstrating the use of genotyping in urban tuberculosis control. BMC Infect Dis 2009;9:151.

\section{Journal club}

\section{Endobronchial valves for advanced emphysema}

In this multicentre randomised trial from USA, the safety and efficacy of unilobar endobronchial valve therapy in patients with heterogeneous emphysema was compared with usual care.

In terms of effectiveness, the co-primary outcomes were percentage change in $\mathrm{FEV}_{1}$ and distance on the $6 \mathrm{~min}$ walk test. In terms of safety, the primary outcome was the difference in complication rate, using a composite of six major complications including death, empyema, massive haemoptysis, pneumonia distal to the valves and pneumothorax or air leak of more than 7 days duration.

Patients with endobronchial valves showed modest improvements in $\mathrm{FEV}_{1}$ and 6 min walk test distance, but at the cost of more pneumonia, including episodes requiring hospitalisation, chronic obstructive pulmonary disease exacerbations and haemoptysis. Follow-up was for 12 months with most complications occurring in the 6 months after valve insertion. There were also modest improvements in secondary end points including quality of life, dyspnoea and supplemental oxygen use.

Of note, there were substantial missing data for the primary efficacy end points, but similar rates were observed in control and intervention groups. There was a higher drop out rate in the control group. It was noted that patients in the high heterogeneity subgroup had greater improvements in $\mathrm{FEV}_{1}$ and distance on the 6 min walk test. The study was not powered to compare subgroups but this suggests that, similar to lung volume reduction surgery, appropriate patient selection is key to good outcomes.

Therapeutic interventions for advanced emphysema are limited. The role of endobronchial valve therapy remains unclear. There are no direct comparisons with lung volume reduction surgery, but benefits are likely to include lower complication rates, and perhaps mortality. Careful patient selection using expert analysis of high-resolution CT is vital and likely to be an area of future research.

Sciurba FC, Ernst A, Herth FJF, et al. A randomized study of endobronchial valves for advanced emphysema. N Engl J Med 2010;363:1233-44.

\section{Laura-Jane E Smith}

Correspondence to Dr Laura-Jane E Smith, ST2, University College Hospital NHS Trust, Department of Thoracic Medicine, 250 Euston Road, London NW1 2PG, UK; smith.laurajane@gmail.com

Published Online First 10 February 2011

Thorax 2011;66:713. doi:10.1136/thx.2011.159442 